\title{
Factores pronósticos del abandono del tratamiento antituberculoso en una región endémica del Perú
}

\author{
Dante Roger Culqui, ${ }^{1}$ Carlos Gabriel Grijalva, ${ }^{1}$ Simy del Rocío \\ Reategui, ${ }^{2}$ Jesús Manuel Cajo ${ }^{2}$ y Luis Antonio Suárez ${ }^{1}$
}

Forma de citar Culqui DR, Grijalva CG, Reategui SR, Cajo JM, Suárez LA. Factores pronósticos del abandono del tratamiento antituberculoso en una región endémica del Perú. Rev Panam Salud Publica. 2005; 18(1):14-20.

RESUMEN Objetivo. Identificar factores de pronóstico del abandono del tratamiento antituberculoso en la provincia de Ica, Perú.

Métodos. Entre 1998 y 2000 se llevó a cabo un estudio de casos y testigos (razón numérica de 1:1) en la provincia de Ica. Se identificaron 55 casos de abandono del tratamiento antituberculoso. Los factores evaluados se seleccionaron a partir del modelo del campo de la salud de Lalonde. Las respectivas razones de posibilidades se calcularon por medio de análisis unifactorial y multifactorial.

Resultados. Se identificaron como factores pronósticos del abandono del tratamiento antituberculoso los siguientes: considerar insuficiente la información proporcionada por el personal de salud sobre el tratamiento (razón de posibilidades [odds ratio, OR]: 4,20; intervalo de confianza de 95\% [IC95\%]: 1,77 a 10,02), considerar inadecuados los horarios para recibir el tratamiento (OR: 9,95; IC95\%: 1,97 a 50,21) y consumir drogas ilícitas (OR: 7,15; IC95\%: $1,69$ a 30,23$)$.

Conclusiones. Para mejorar el cumplimiento del régimen antituberculoso es necesario brindar a los pacientes información personalizada sobre la enfermedad y su tratamiento, además de ofrecerles horarios flexibles y apropiados para recibirlo. El consumo de drogas es el factor de riesgo más alto de abandono, por lo que resultan cruciales su identificación y seguimiento.

Palabras clave Rechazo al tratamiento, tuberculosis, Perú.

1 Oficina General de Epidemiología. Ministerio de Salud del Perú. Toda correspondencia deberá dirigirse a: Dante Roger Culqui, Oficina General de Epidemiología, Ministerio de Salud. (OGEMINSA), Jr. Camilo Carrillo 402, Jesús María, Lima, Lima 11, Perú. Tel. 433 5859, anexo 20. Correo electrónico: dculqui@hotmail.com

2 Facultad de Medicina, Universidad Nacional San Luis Gonzaga en Ica. Ica, Perú.
La tuberculosis, que es la principal causa de muerte por enfermedades infecciosas en adultos y ocasiona $1,9 \mathrm{mi}$ llones de muertes cada año en el mundo, se presenta con una frecuencia anual aproximada de 8 millones de casos nuevos. La Organización Mundial de la Salud ha estimado que, de no emprenderse medidas de control adi- cionales, para el año 2020 el número de casos nuevos se habrá elevado a $10 \mathrm{mi}$ llones (1-4). En América del Sur, el mayor número de casos y las tasas más altas de incidencia se concentran en Brasil y Perú $(2,5)$. En 1999, con una incidencia estimada de 228 por 100000 habitantes, Perú era uno de los 23 países del mundo en que se concentra- 
ban $80 \%$ de los casos nuevos de tuberculosis. A pesar de que la población del país representa apenas 3\% de la población del continente americano, para esa fecha Perú reunía 15\% de los casos de tuberculosis registrados en este territorio $(2,6)$.

Las actividades que se han emprendido en el Perú para el control de la tuberculosis, basadas en la estrategia de tratamiento breve bajo observación directa (directly observed treatment-short course, DOTS), recibieron el reconocimiento de la Organización Mundial de la Salud en el año 2001. Entre los países con una alta carga de tuberculosis, para esa fecha solo Vietnam y Perú habían alcanzado los objetivos de una detección de $70 \%$ de los casos y la cura de $85 \%$ de ellos (4-7).

El abandono del tratamiento antituberculoso, que el Ministerio de Salud del Perú define como "no concurrir a recibir el tratamiento por más de treinta días consecutivos" (8), es la principal limitación para lograr la cura de esta enfermedad. Además de que impide la curación, el abandono guarda una relación estrecha con una pronta recaída y, no obstante ser una causa reversible, constituye el mayor factor determinante del fracaso terapéutico. Es también un antecedente común en pacientes que mueren de tuberculosis $(5,9-12)$.

El tratamiento antituberculoso es un proceso complejo que requiere un régimen terapéutico prolongado (de meses). Durante ese proceso, diversos factores pueden influir en la decisión de continuarlo o abandonarlo (13); destacan los factores relacionados con los servicios y el personal de salud, además de otros propios de los pacientes y del ambiente que los rodea. Es fundamental que los servicios de salud ofrezcan gratuitamente los medicamentos, realicen un seguimiento por medio de visitas domiciliarias periódicas y brinden al paciente información adecuada y suficiente acerca de la enfermedad y el tratamiento (14, 15). Por otra parte, la baja escolaridad, la incertidumbre de si se logrará la cura al final del tratamiento y la falta de apoyo familiar, aunadas a la impo- sibilidad de faltar al trabajo para acudir a la consulta terapéutica, de cumplir con el horario impuesto por el centro de salud y de costear el transporte necesario, son factores que pueden influir negativamente en la continuidad del régimen especifico. Otros factores que se sabe incrementan la probabilidad de abandono son el estigma social de la enfermedad, las reacciones adversas o la intolerancia a los medicamentos y la adicción al alcohol o a las drogas (13).

Situada en la costa peruana, la provincia de Ica es una región gravemente afectada por la enfermedad que nos ocupa. Presenta una de las mayores tasas de incidencia en el país y concentra una elevada proporción de abandonos del tratamiento antituberculoso (8). En 1998, la incidencia de abandono alcanzó $5,6 \%$, cifra que rebasa el valor que establece como límite el programa de control de la tuberculosis (PCT) (5\% de abandonos). En los últimos años se ha atestiguado una elevación progresiva de la frecuencia de abandonos, con valores de 5,7 y 5,8\% para los años 2002 y 2003, respectivamente. ${ }^{3}$

El objetivo de la presente investigación ha sido identificar los factores pronósticos e indicadores del riesgo de abandono del tratamiento antituberculoso que incidieron en la provincia de Ica, Perú, durante los años de 1998 a 2000. La identificación de estos factores e indicadores permitirá emprender medidas que incrementen la eficacia de las actividades de control de la enfermedad.

\section{MATERIAL Y MÉTODOS}

Durante el año 2001 se realizó un estudio de casos y testigos que incorporó a los pacientes inscritos para tratamiento antituberculoso en el programa de control de la tuberculosis de la provincia de Ica, entre el $1^{\circ}$ de enero de 1998 y el 31 de diciembre de 2000.

\footnotetext{
3 Datos no publicados. Programa de Control de Tuberculosis, Dirección Regional de Salud de Ica.
}

Se definió como caso a todo paciente que hubiera abandonado el tratamiento por un período mínimo de 30 días consecutivos. De la misma forma, se consideró testigo todo aquel que recibió el régimen terapéutico antituberculoso y terminó el tratamiento en el tiempo establecido por el programa de control de la tuberculosis.

La selección se basó también en los siguientes criterios de exclusión:

- pacientes que no eran residentes de la provincia de Ica, transferidos de otras regiones, transferidos a otras regiones y aquellos sin domicilio identificable

- pacientes con el antecedente de abandono del tratamiento

- pacientes fallecidos durante el período de estudio

- niños menores de 7 años, porque su tratamiento no incluye el etambutol, medicamento común en los esquemas para enfermos de mayor edad

- pacientes que el PCT considera "irregulares" en el tratamiento. Se consideró "irregular" a aquel que, si bien no satisface la definición de abandono, muestra falta de continuidad en el tratamiento. Esta condición puede hacer que el régimen se extienda semanas o meses (hasta un máximo de 70 días adicionales al esquema inicial) (8).

Las variables explicativas se definieron conforme al modelo epidemiológico de los componentes del campo de la salud de Laframboise y Lalonde, el cual tiene en cuenta la biología humana, el modo de vida, el ambiente y los servicios de salud (16). Entre los factores propios de la biología humana se consideraron características como sexo, edad, la sensación de mejoría durante el tratamiento y la aparición de reacciones adversas durante este período. Entre los factores relacionados con el ambiente se consideraron el grado de instrucción, el estado civil, la presencia de temores o creencias respecto al tratamiento y la enfermedad, la pobreza, el entorno fa- 
miliar y laboral, la disponibilidad de tiempo para acudir al tratamiento y la accesibilidad geográfica del lugar donde este se administra. Entre las características relacionadas con los establecimientos de salud, se evaluó el conocimiento del paciente acerca de su enfermedad y tratamiento, la percepción de los peligros de abandonarlo, la satisfacción con el servicio prestado y el seguimiento del caso durante el tratamiento. Entre las variables relacionadas con el modo de vida, se prestó particular atención a la presencia de hábitos nocivos, como el consumo de alcohol, tabaco y drogas. Se consideró consumo frecuente de cigarrillos el de un cigarrillo cada tercer día en promedio, o más de cuatro a la semana. El consumo frecuente de alcohol se definió como el consumo de bebidas alcohólicas hasta alcanzar la embriaguez por lo menos una vez por semana. Y el consumo de drogas ilícitas, como cualquier uso de estas sustancias, independientemente de la frecuencia o tipo de droga.

\section{Análisis estadístico}

Las variables categóricas entre casos y testigos se analizaron mediante una prueba de ji al cuadrado. El análisis bifactorial proporcionó la razón de posibilidades (odds ratio, RP) bruta correspondiente, y las variables relacionadas con el desenlace (abandono del tratamiento antituberculoso) y posibles factores de confusión se ingresaron en un modelo multifactorial de regresión logística. Se evaluaron de acuerdo con la prueba de máxima verosimilitud en modelos anidados sucesivos, en los cuales las covariables se ajustaron de acuerdo con el número de valores perdidos y los grados de libertad en cada paso. Se consideraron estadísticamente significativos los resultados de pruebas con un valor $P$ menor de 0,05 y se calcularon los intervalos de confianza de 95\% (IC95\%) de cada una de las estimaciones. Para el análisis estadístico se empleó el programa informático Stata 8,2 (Stata Corporation, College Station, Texas, Estados Unidos).

\section{RESULTADOS}

En su mayoría, los pacientes que recibían tratamiento antituberculoso fueron del sexo masculino. Las proporciones de los que manifestaron molestias adicionales durante el tratamiento fueron semejantes entre casos y testigos. Asimismo, la proporción de los que refirieron no tener otros síntomas durante el régimen fue semejante en ambos grupos. También la procedencia de áreas rurales fue similar en ambos grupos de pacientes. El análisis bifactorial no reveló diferencias significativas entre casos y testigos cuando se compararon las proporciones de sujetos que refirieron vivir a más de 30 minutos de los establecimientos de salud según su medio de transporte usual. Sin embargo, la proporción de los que consideraron excesivo el gasto de trasladarse al establecimiento de salud para recibir tratamiento fue tres veces mayor entre los casos que en el grupo testigo. En el cuadro 1 se resumen las características de los sujetos de estudio, de acuerdo con el modelo epidemiológico utilizado.

La escolaridad menor que instrucción secundaria completa se relacionó con una mayor probabilidad de renunciar al tratamiento $(P=0,001)$. De igual manera, una mayor proporción de casos que de testigos refirió tener una relación familiar conflictiva $(P=0,014)$. Por lo contrario, las proporciones de sujetos que dijeron profesar una religión, tener miedo del tratamiento y creer que los remedios caseros pueden curar la enfermedad, fueron semejantes en ambos grupos.

Con respecto a los servicios de salud, la opinión de que los horarios establecidos para recibir la medicación eran inadecuados se relacionó con una mayor probabilidad de abandono $(P=$ $0,001)$. De la misma forma, la proporción de casos que declaró estar disconforme con el trato del personal de salud fue tres veces superior a la de los testigos. En ambos grupos, cerca de $30 \%$ de los sujetos opinaron que el tiempo de espera para recibir su medicación era excesivo. En comparación con los testigos, un mayor porcentaje de casos declaró disconformidad con la duración del tratamiento $(P=0,033)$. La mayoría de los pacientes de ambos grupos expresó disconformidad con la cantidad de medicamentos utilizados, que en su opinión era excesiva. La declaración de no contar con tiempo suficiente para acudir a tratamiento fue significativamente más frecuente entre los casos que entre los testigos $(P=$ $0,032)$. Proporciones semejantes de pacientes de ambos grupos consideraron que el tratamiento no los curaría. Los que lo abandonaron desconocían la duración de su propio régimen con mayor frecuencia que los testigos $(P=$ $0,005)$, En ambos grupos, una menor proporción desconocía el número de pastillas que debía tomar cada día.

Proporciones semejantes de casos y testigos señalaron desconocer los riesgos de renunciar el tratamiento específico. Del mismo modo compartieron la opinión de que el personal que los tenía a cargo no se encontraba debidamente capacitado. Hasta $65 \%$ de los casos consideraron insuficiente la información que ofrece el programa, mientras que la proporción fue significativamente menor en el grupo testigo $(P=0,001)$. Más de $50 \%$ de los casos no conocían el esquema terapéutico que estaban recibiendo y una proporción semejante se observó en el grupo testigo.

Cuando dejaron de asistir a tratamiento, $19 \%$ de los casos refirieron no haber recibido la correspondiente visita domiciliaria del personal del programa, frente a una proporción significativamente mayor entre los testigos $(P=0,004)$.

En cuanto a los hábitos nocivos, proporciones semejantes refirieron consumo frecuente de tabaco en ambos grupos. El consumo frecuente de alcohol y drogas mostró relación significativa con una mayor probabilidad de abandonar el tratamiento antituberculoso $(P=0,042$ y $P=0,006$, respectivamente).

Cuando se controló simultáneamente el efecto de otras variables en el análisis multifactorial, se identificó como factor de riesgo de abandono la opinión de que la información proporcionada por el programa era deficiente $(P=0,025)$, lo mismo que el concepto 
CUADRO 1. Características de los sujetos, por componentes del campo de la salud. Ica, Perú, 1998-2000

\begin{tabular}{|c|c|c|c|c|c|}
\hline & \multicolumn{2}{|c|}{ Testigos $(n=59)$} & \multicolumn{2}{|c|}{ Casos $(n=58)$} & \multirow[b]{2}{*}{$P$} \\
\hline & No. & $\%$ & No. & $\%$ & \\
\hline \multicolumn{6}{|l|}{ Biología humana } \\
\hline No sintió mejoría durante el tratamiento & 4 & 6,78 & 7 & 12,07 & 0,327 \\
\hline Permaneció asintomático durante el tratamiento & 24 & 40,68 & 20 & 34,48 & 0,489 \\
\hline \multicolumn{6}{|l|}{ Ambiente } \\
\hline Considera excesivo el gasto para acudir al tratamiento & 3 & 5,08 & 9 & 15,52 & 0,063 \\
\hline Se considera practicante de alguna religión & 57 & 96,61 & 55 & 94,83 & 0,634 \\
\hline Cree que los remedios caseros curan la tuberculosis & 16 & 27,12 & 22 & 37,93 & 0,212 \\
\hline Tiene temor al tratamiento & 25 & 42,37 & 24 & 41,38 & 0,913 \\
\hline Refiere tener una relación familiar conflictiva & 2 & 3,39 & 10 & 17,24 & 0,014 \\
\hline Vive lejos del establecimiento de salud (más de $30 \mathrm{~min}$ ) & 12 & 20,34 & 18 & 31,03 & 0,185 \\
\hline No está conforme con la duración del tratamiento & 25 & 42,37 & 36 & 62,07 & 0,033 \\
\hline Considera excesiva la cantidad de medicamentos que recibe & 35 & 59,32 & 41 & 70,69 & 0,198 \\
\hline No tuvo el tiempo necesario para acudir al tratamiento & 7 & 11,86 & 16 & 27,59 & 0,032 \\
\hline No cree que el tratamiento lo curará & 5 & 8,47 & 9 & 15,52 & 0,241 \\
\hline No sabe cuánto dura el tratamiento & 4 & 6,78 & 15 & 25,86 & 0,005 \\
\hline No sabe cuántas pastillas debe tormar al día & 8 & 13,56 & 12 & 20,69 & 0,306 \\
\hline Refiere no conocer el riesgo de abandonar el tratamiento & 12 & 20,34 & 16 & 27,59 & 0,358 \\
\hline Considera deficiente la información que le da el programa & 20 & 33,90 & 38 & 65,52 & 0,001 \\
\hline Considera que el personal no está debidamente capacitado & 18 & 30,51 & 19 & 32,75 & 0,794 \\
\hline No sabe qué esquema de tratamiento recibe & 30 & 50,85 & 35 & 60,34 & 0,301 \\
\hline $\begin{array}{l}\text { No recibió visita del progarma al faltar por tercer día } \\
\text { al tratamiento }\end{array}$ & 26 & 44,07 & 11 & 18,97 & 0,004 \\
\hline \multicolumn{6}{|l|}{ Estilo de vida } \\
\hline
\end{tabular}

de que los horarios del programa eran inadecuados $(P=0,025)$. Por su parte, el consumo de drogas mostró relación significativa con este riesgo $(P=0,047)$ (cuadro 2 y figura 1).

\section{DISCUSIÓN}

El abandono del tratamiento antituberculoso es uno de los principales problemas para el control de la tuberculosis y la principal causa de recaídas y de surgimiento de cepas resistentes $(11,12)$. El presente estudio pone de manifiesto una firme relación entre considerar deficiente la información que proporciona el personal de salud y el abandono del tratamiento antituberculoso. La estrategia DOTS, adoptada en los establecimientos de salud, requiere, además de la administración supervisada de los medicamentos, brindar a cada paciente la información apropiada siguiendo un criterio personalizado, al tiempo que se promueven el reforzamiento y la motivación para proseguir el régimen. En este contexto, el proveedor de salud está en situación ideal para identificar cualquier intento de abandono o interrupción del esquema terapéutico y, de esa forma, intervenir oportunamente $(3,13,17)$. De Lima et al. destacaron la presencia de estas condiciones positivas en pacientes con buena adherencia al tratamiento específico (18). Asimismo, Orozco et al. identificaron que la adecuada información proveída por el personal de salud reduce la probabi- lidad de que el paciente abandone el tratamiento (19). Por otra parte, el desconocimiento o la falta de buena información sobre la enfermedad se ha relacionado con mayor riesgo de deserción (20).

Otro importante factor de riesgo de abandono del tratamiento antituberculoso es la apreciación de que los horarios establecidos para su administración son inadecuados. Además, dado que el tratamiento bajo observación directa requiere el desplazamiento del paciente hacia el establecimiento de salud, con la consiguiente inversión de tiempo y dinero, es muy probable que a muchos de ellos les resulte incómodo este esquema, de larga duración (21, 22). Si bien la tuberculosis se ha asociado habitualmente con las poblacio- 
CUADRO 2. Factores e indicadores del riesgo de abandono del tratamiento antituberculoso. Ica, Perú, 1998-2000

\begin{tabular}{|c|c|c|c|c|c|c|c|c|}
\hline \multirow{2}{*}{ Ambiente } & \multicolumn{4}{|c|}{ Análisis unifactorial } & \multicolumn{4}{|c|}{ Análisis multifactorial } \\
\hline & $\mathrm{RP}$ & \multicolumn{2}{|c|}{ IC95\% } & $P$ & $\mathrm{RP}$ & \multicolumn{2}{|c|}{ IC95\% } & $P$ \\
\hline Considera excesivo el gasto para acudir al tratamiento & 3,43 & 0,79 & 20,57 & 0,063 & 4,60 & 1,04 & 20,36 & 0,044 \\
\hline Refiere tener una relación conflictiva en casa & 5,94 & 1,17 & 57,54 & 0,014 & 4,22 & 0,73 & 24,36 & 0,107 \\
\hline \multicolumn{9}{|l|}{ Servicios de salud } \\
\hline No está conforme con la duración del tratamiento & 2,23 & 1,00 & 4,99 & 0,033 & 1,46 & 0,61 & 3,51 & 0,400 \\
\hline No tuvo el tiempo necesario para acudir al tratamiento & 2,83 & 0,98 & 8,85 & 0,032 & 1,07 & 0,32 & 3,62 & 0,911 \\
\hline Refiere no conocer la duración del tratamiento & 4,80 & 1,38 & 21,02 & 0,005 & 2,83 & 0,73 & 11,03 & 0,134 \\
\hline \multicolumn{9}{|l|}{ Modo de vida } \\
\hline Consume alcohol & 2,27 & 0,96 & 5,46 & 0,042 & 1,93 & 0,76 & 4,92 & 0,170 \\
\hline Consume drogas ilícitas & 5,39 & 1,35 & 30,87 & 0,006 & 7,15 & 1,69 & 30,23 & 0,007 \\
\hline
\end{tabular}

nes de menor poder económico $(3,11$, 12), el nivel socioeconómico no sería un factor determinante en el abandono del régimen (23). En ese contexto, el horario de atención de los establecimientos de salud suele coincidir con el horario de la mayor parte de las actividades laborales, lo que implicaría, en el caso de los pacientes que trabajan, una frecuente ausencia temporal de sus centros de trabajo, con los riesgos, temores y consecuencias negativas que esto puede acarrear. $\mathrm{Al}$ evaluar los retrasos o problemas que se susciten en un establecimiento de salud en relación con el horario de administración del tratamiento específico, se deben ponderar estos factores, a fin de no influir negativamente en el curso del tratamiento.

Los resultados de este estudio ponen de manifiesto una relación significativa entre el consumo de drogas ilícitas y la probabilidad de abandono del tratamiento antituberculoso. Si bien esa relación ya se ha señalado en otros escritos, en muy pocos casos se ha establecido el diagnóstico de drogadicción, en contraposición al consumo frecuente de drogas, especialmente cuando el consumo incluye drogas endovenosas $(11,13,14,22,24)$. Este importante indicador de riesgo permite identificar un grupo específico al que se debe prestar especial atención en el transcurso del régimen terapéutico.

A pesar de que estudios previos relacionan la baja escolaridad con una mayor probabilidad de abandono del tratamiento antituberculoso $(20,25$, 26), el presente análisis no revela una relación significativa entre tener una instrucción inferior a la secundaria y el desenlace que se evalúa. Los autores consideran que, más que la escolaridad del individuo, en la decisión de proseguir o dejar el tratamiento influyen la información que el paciente recibe sobre su enfermedad y los métodos para tratarla y el entorno del establecimiento de salud al que acude.

FIGURA 1. Factores pronósticos del abandono del tratamiento antituberculoso en la provincia de Ica, Perú, 1998-2000

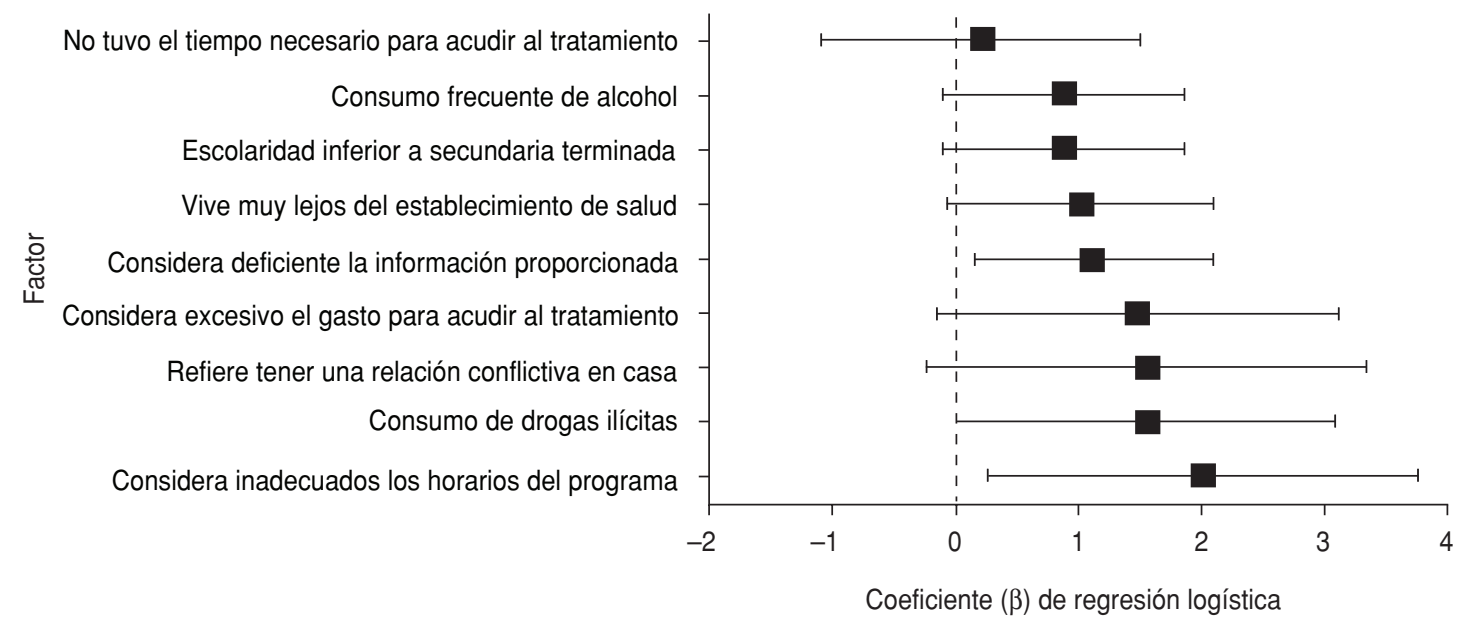


Estudios anteriores han señalado una fuerte asociación entre el alcoholismo y el abandono del tratamiento tuberculoso (19, 27-29). Aunque en nuestra población de estudio el consumo de alcohol fue significativamente más alto en los casos que en los testigos, una vez realizado el ajuste respecto a otras variables, dicha asociación dejó de ser estadísticamente significativa. Un dato interesante del estudio es que el consumo referido de alcohol fue alto tanto en los casos como en los testigos (24 y $42 \%$ respectivamente), lo que quizá contribuyó a atenuar la asociación entre esta variable y el desenlace.

Se ha sugerido influencia del entorno familiar en la continuidad del tratamiento, en el sentido de que una relación conflictiva puede favorecer el abandono (30). Sin embargo, cuando los datos se ajustaron respecto a otras variables, dicha asociación no conservó su significación estadística. Mejía et al. (20), utilizando el indicador "grado de apoyo familiar" como factor individual relacionado con el abandono, tampoco encontraron asociación significativa. En este sentido, la atención, información y facilidades que pueden brindarse al paciente en el establecimiento de salud son factores que tienen mayor repercusión en la continuidad del tratamiento.
$\mathrm{Al}$ interpretar estos resultados deben considerarse las limitaciones del presente estudio. La recopilación de hechos que ocurrieron en el pasado siempre está expuesta al sesgo de memoria. También el conocimiento a priori del tipo de participante (caso o testigo) pudo introducir sesgos en el personal que participó en la recopilación de datos. Además, el tamaño limitado de la población de estudio puede restringir la potencia del análisis multifactorial para evaluar asociaciones e interacciones de interés, al ingresar simultáneamente varias covariables en el modelo. Por otra parte, algunas condiciones de interés, en particular el consumo de tabaco, alcohol y drogas, se evaluaron mediante preguntas sencillas cuyas respuestas pudo manipular el entrevistado, ya sea para minimizar $\mathrm{o}$, menos probablemente, magnificar la situación real. Por otra parte, el estudio no evaluó la presencia de adicción a alguna de estas sustancias, lo cual habría requerido la intervención de personal especialmente capacitado para ese fin. Sin embargo, los factores identificados en el presente análisis son congruentes con la mayor parte de los estudios publicados sobre el tema. Nuestros resultados apuntan a que muchos de los mismos factores de riesgo de abandono del tratamiento antituberculoso que se observan en otras regiones se presentan también en la provincia de Ica, Perú. Las iniciativas para el control de la tuberculosis están sometidas a un proceso constante de cambios y mejoras. En ese sentido, los resultados presentes son compatibles con la necesidad cada vez más evidente de realizar investigaciones que se enfoquen en los complejos factores que inciden en la conducta de los pacientes en tratamiento antituberculoso. La identificación individualizada de dichos factores, articulada con una intervención apropiada y oportuna, reduciría en grado importante las tasas de abandono del tratamiento antituberculoso.

\section{CONCLUSIONES}

Sin duda, no son problemas de fácil solución el consumo de drogas, la información deficiente que reciben los pacientes tuberculosos del personal de salud ni la inversión de tiempo y dinero que requiere el tratamiento supervisado. Sin embargo, la identificación de estos factores e indicadores de riesgo permitirá comprender mejor la compleja problemática del abandono del tratamiento antituberculoso y ayudará a orientar las actividades de control de la tuberculosis.

\section{REFERENCIAS}

1. World Health Organization. Global Tuberculosis Control: Surveillance, Planning, Financing. WHO Report 2002. Ginebra: WHO; 2002. (WHO/CDS/TB/2002.295)

2. Dye C, Scheele S, Dolin P, Pathania V, Raviglione M. Global burden of tuberculosis: estimated incidence, prevalence and mortality by country. JAMA. 1999;282:677-86.

3. World Health Organization. An Expanded DOTS Framework for Effective Tuberculosis Control. Ginebra: WHO; 2002. (WHO/CDS/ $\mathrm{TB} / 2002.297$ ).

4. Wise J. WHO identifies 16 countries struggling to control tuberculosis. Br Med J. 1998; 316:955.

5. Meza M, Accinelli R, Mendoza D. Factores de riesgo para el fracaso del tratamiento antituberculoso totalmente supervisado. Rev Sociedad Peruana Med Interna. 1999; 33(8):140-4.

6. Suárez P, Watt C, Alarcón E, Portocarrero J, Zavala D, Canales $R$ et al. The dynamics of tuberculosis in response to 10 years of intensive 1994:330(17):1179-84. control effort in Peru. J Infect Dis. 2001;184: 473-8.

7. World Health Organization. Global tuberculosis control. WHO Report 2001. Ginebra: WHO; 2001. (WHO/CDS/TB/2001.287).

8. Programa Nacional de Control de Enfermedades Transmisibles-Control de la Tuberculosis. Lima: Ministerio de Salud del Perú; 2000.

9. Mendoza D, Benites C, Matzuoka G, Meza M. Eficacia y eficiencia del programa de control de tuberculosis en Rioja, San Martín, Perú, durante el período de 1996-2000. Rev Med Hered. 2001; 18(8):14-20.

10. García M, Mayar M, Ferreira L, Palacios M. Eficacia y eficiencia del tratamiento antituberculoso en jurisdicciones sanitarias de Morelos, México. Salud Publica Mex. 1998;40(5):421-9.

11. Weis SE, Slocum PC, Blais FX, King B, Nunn $M$, Matney GB et al. The effect of directly observed therapy on the rates of drug resistance and relapse in tuberculosis. N Engl J Med.
12. Anibarro L, Lires JA, Iglesias F, Vilariño $C$, Baloria A, de Lis JM et al. Factores sociales de riesgo para la falta de cumplimiento terapéutico en pacientes con tuberculosis en Pontevedra. Gac Sanit. 2004;18(1):38-44.

13. Ribeiro SA, Amado VM, Camelier AA, et al. A case-control study about indicators of noncompliance in patients with tuberculosis. J Pneumol. 2000;26(6):291-6.

14. Wallace Fox. Compliance of patients and physicians: experience and lessons from tuberculosis-II. Br Med J. 1993;287:101-5.

15. Wallace Fox. Compliance of patients and physicians: experience and lessons from tuberculosis-I. Br Med J. 1993;287:33-5.

16. Dever AG. Epidemiología y administración de los servicios de salud. Washington, D.C. Organización Panamericana de la Salud; 1991.

17. Volmink J, Garner P. Systematic review of randomized controlled trials of strategies to promote adherence to tuberculosis treatment. Br Med J. 1997;315:1403-6. 
18. De Lima MB, Mello DA, Morais AP, da Silva WC. Estudo de casos sobre abandono do tratamento da tuberculose: avaliação do atendimento, percepção e conhecimentos sobre a doença na perspectiva dos clientes (Fortaleza, Ceará, Brasil). Cad Saude Publica. 2001;17(4): 877-85.

19. Orozco L, Hernández R, Usta C. Factores de riesgo para el abandono (no adherencia) del tratamiento antituberculoso. Medicas UIS (Revista de los Estudiantes de Medicina de la Universidad Industrial de Santander). 1998; 12(4)169-72.

20. Mejía J, Carmona E. Abandono del tratamiento antituberculoso: factores asociados. Revista Médica del Instituto Mexicano del Seguro Social. 1995;33(5):481-5.

21. García-García ML, Mayar-Maya ME, FerreyraReyes L, Palacios-Martínez M, Álvarez-García C, Valdespino-Gómez JL. Efficacy and efficiency of anti-tuberculosis treatment in 2 sanitary jurisdictions of Morelos, Mexico. Salud Publica Mex. 1998;40:421-9.

22. Pablos-Méndez A, Knirsch CA, Graham Barr $\mathrm{R}$, Lerner BH, Frieden TR. Nonadherence in tuberculosis treatment: predictors and consequences in New York City. Am J Med. 1997; 102:164-70.

23. Sbarbaro JA. Public health aspects of tuberculosis: supervision of therapy. Clin Chest Med. 1980;1:253-63.

24. Delgado R, et al. Causas de abandono al tratamiento de los pacientes de PCT del centro de salud "El Progreso". [Tesis para obtener el grado de Bachiller en Medicina Humana.] Universidad Peruana Cayetano Heredia. Lima; 1985.

25. Yactayo SD. Causas de abandono del tratamiento antituberculosis en Lima. Tesis para obtener el grado de Bachiller en Medicina. Universidad Peruana Cayetano Heredia. Lima; 1992.

26. Manjarrez Morales EM, Serrano MV, Cano PG, Verduzco GE, Escandon RC, Escobedo DLP. [Main causes of non-compliance with pulmonary tuberculosis treatment.] Gac Med Mex. 1993;129(1):57-62.

27. Córdova R, Pacheco D. Factores que influyen en el abandono del programa de control de tuberculosis en algunos centros de salud de
Arequipa. [Tesis para obtener el grado de Bachiller en Medicina.] Arequipa: Universidad Nacional de San Agustín; 1996.

28. De Fatima Militão $M$, de Sá CC, Leite $A R$ Vieira W, Salustiaño A. Fatores prognósticos para o desfecho do tratamento da tuberculose pulmonar em Recife, Pernambuco, Brasil. Rev Panam Salud Publica. 2001;9(6):368-74.

29. Ferrer S, Galvano XK. Caracterización del paciente tubeculoso que abandona el tratamiento. [Tesis para obtener el grado de Bachiller en Medicina.] Concepción (Chile): Universidad de Concepción; 1997.

30. Lengua RG. Factores socio-demográficos culturales que influyen en el abandono al tratamiento de tuberculosis de los pacientes en el Centro de Salud "El Progreso", Lima 19951996. [Tesis para obtener el grado de Licenciado en Enfermería.] Lima: Universidad Peruana Cayetano Heredia; 1998.

Manuscrito recibido el 21 de septiembre de 2004. Aceptado para publicación, tras revisión, el 9 de marzo de 2005.

ABSTRACT Objective. To identify factors that predict noncompliance with tuberculosis treatment in the province of Ica, Peru.

\section{Predictive factors for noncompliance with tuberculosis treatment in an endemic region of Peru}

Methods. Between 1998 and 2000 a case-control study (1:1 ratio) was conducted in the province of Ica, with 55 cases (persons who dropped out of treatment) being identified. The factors evaluated were chosen from Lalonde's model of the field of health. The respective odds ratios were calculated by means of univariate analysis and multivariate analysis.

Results. The following factors were identified as being predictive of noncompliance with tuberculosis treatment: thinking that the information that health workers provided on treatment was insufficient (odds ratio $(O R)=4.20 ; 95 \%$ confidence interval (95\% CI), 1.77-10.02), thinking that the office hours for receiving treatment were inadequate $(\mathrm{OR}=9.95 ; 95 \% \mathrm{CI}, 1.97-50.21)$, and consuming illegal drugs $(\mathrm{OR}=7.15$; 95\% CI, 1.69-30.23).

Conclusions. To improve compliance with tuberculosis treatment, it is necessary to provide patients with personalized information on the disease and its treatment, and to offer them flexible, appropriate times to receive treatment. Drug consumption is the most serious risk factor for noncompliance, and identifying and following up with drug users is crucial.

Keywords Treatment refusal, tuberculosis. 